\title{
Simplified Residual Phase Correction Mechanism for the IEEE 802.11a Standard
}

\author{
Alfonso Troya, Miloš Krstić, Koushik Maharatna \\ IHP \\ Im Technologiepark 25 \\ 15236 Frankfurt (Oder), Germany \\ E-mail: \{troya, krstic, maharatna\}@ihp-microelectronics.com
}

\begin{abstract}
It is of common use in OFDM transmission to define some of the sub-carriers as pilots. These sub-carriers contain information, which is known a priori at the receiver and are typically used for channel estimation. In the special case of the IEEE 802.11a standard, the pilots cannot be used for channel estimation because of their wide separation in the frequency domain. However they are useful to track phase variations remaining after synchronization during reception of a frame. In this paper we propose a very simple mechanism to estimate and correct these phase variations using the pilots. We also present a channel estimator necessary to support such a simplified mechanism.
\end{abstract}

Keywords-Pilot-assisted OFDM; synchronization; channel estimation; phase correction

\section{INTRODUCTION}

OFDM signals are very much sensitive to the synchronizer performance, mainly because the different sub-carriers overlap their respective spectra. The synchronizer is the block responsible for detecting the incoming frame and to estimate and correct for possible frequency offsets. It also identifies the starting point from which on the different OFDM symbols will be fed into the FFT block.

To carry out all the operations mentioned above, the standard IEEE 802.11a [1] defines the so-called preamble symbols, which have a very specific structure to simplify the estimation procedures. A suitable synchronizer architecture for this standard was presented by the authors in [2].

Nevertheless, the synchronizer cannot compensate for some undesirable effects prompted by the RF downconversion and the analog-to-digital converters. Furthermore, the synchronizer itself will introduce some estimation errors. Speth [3] and Robertson [4] showed that all these impairments are visible inside an OFDM symbol as a linear phase.

Pilot sub-carriers inside an OFDM symbol may help to easily estimate the remaining linear phase if they were used during the channel estimation, because this phase would be seen as a part of the channel itself. For the IEEE 802.11a standard, the pilots are not intended for channel estimation, i.e. the remaining linear phases should be explicitly estimated using the pilots. A straightforward solution requires an arctangent operation to estimate the linear phase, together with an NCO to correct for it. Both operations may be realized using computationally intensive algorithms. In the solution we propose here, the linear phase is reduced to a nearly constant phase throughout the OFDM symbol and thus, it allows a significant simplification of the whole operation.

The paper is organized as follows: in Section II, the main contributors to the linear phase are discussed. Section III introduces a decision-directed channel estimator, firstly proposed by Mignone in [5], which will be the basis for our simplified algorithm. In Section IV, the residual phase estimation and correction mechanism is investigated and Section $\mathrm{V}$ is devoted to the presentation of some simulation results to support the validity of this method. Finally, some conclusions are derived in Section VI.

\section{CONTRIBUtors to THE Residual Phase}

\section{A. Erroneous Frame Timing Estimation}

During synchronization the symbol timing has to be estimated for proper data decoding. The resolution in the determination of this timing will strongly depend on the sampling interval, i.e. the system is limited to estimate timing errors, which are a multiple of this sampling period. Therefore, the uncertainty in the estimation will be $\pm 0.5 \mathrm{~T}_{\mathrm{S}}$, where $\mathrm{T}_{\mathrm{S}}$ is the sampling period. As explained in [3], a timing error will be seen as a linear phase error after performing the FFT. This phase error will be the same for all the OFDM symbols in that frame. In addition to this, an Inter-Carrier Interference (ICI) component may appear due to the lost of orthogonality, thus increasing the noise content inside the OFDM symbol.

\section{B. Erroneous Carrier Frequency Estimation}

In a typical practical scenario, during RF down-conversion, the different oscillators are not exactly tuned to the expected frequencies. The preamble symbols are used to estimate the carrier frequency offset, as mentioned before. Nevertheless, thermal noise as well as digital noise will affect the estimation. A good synchronizer estimates the frequency offset within an error of $\pm 0.1 \%$. This small residual carrier frequency error generates a constant phase inside the OFDM symbol after FFT, [3]. However, unlike the case of the timing error, this 
phase gets accumulated from symbol to symbol, thus becoming a large phase after several symbols. Furthermore, ICI will occur due to the lost of orthogonality.

\section{Phase Noise}

The several PLLs used during RF down-conversion will generate phase noise. There are mainly two effects associated with the phase noise: Inter-Carrier Interference (ICI) and Common Phase Error (CPE). Interestingly, both ICI and CPE depend on the number of sub-carriers $\mathrm{N}$ used by the OFDM system but in an inverse way. The bigger $\mathrm{N}$, the greater is the ICI power, but the smaller the CPE, and vice versa [4].

The ICI appears as an additive Gaussian noise and the only way to combat it is by redesigning the RF oscillators. The CPE is seen as a constant phase inside the OFDM symbol, similar to the effect mentioned above when a residual carrier frequency offset is present. Nevertheless, in this case the constant phase is not getting accumulated, but changes randomly from symbol to symbol. Unfortunately, the phase affecting one symbol is very little correlated to the ones affecting previous symbols. Therefore, no estimation method based on any linear prediction will give good results. In the IEEE 802.11a, 64 subcarriers are used, and the CPE will hence be much more dominant than the ICI. This is different for other OFDM systems like DAB or DVB, where thousands of sub-carriers are used.

\section{Sampling Clock Frequency Error}

In a real implementation, the system is designed to sample the analog input signal at a certain frequency $\left(f_{\mathrm{S}}\right)$. However, the oscillator will introduce some error in $f_{\mathrm{S}}$. In the IEEE 802.11a standard, 80 samples per symbol are expected, before the FFT and cyclic prefix extraction, with $f_{\mathrm{S}}=20 \mathrm{MHz}$. In the case of a sampling oscillator with e.g. $20 \mathrm{ppm}$ frequency error, this turns into $f_{\mathrm{S}}=20000400 \mathrm{~Hz}$. Thus, 80.00002 samples are obtained for the initial symbol instead of exactly 80 , i.e. a timing error of 0.00002 samples. This timing error is not fixed, but it will be 0.00004 samples for the next symbol, 0.00006 for the third one and so on. In essence, the sampling clock frequency error will be seen as a dynamic timing error. Previously it has been explained that a timing error generates a linear phase error after FFT. In this case the slope of this linear phase error will change from symbol to symbol.

\section{E. Combination of Errors}

As a result, sub-carrier $k$ belonging to symbol $i$ can be expressed as follows after FFT calculation

$$
\mathrm{Y}(i, k)=\mathrm{H}(i, k) \cdot \mathrm{A}(i, k) \cdot e^{j \cdot \phi_{i}(k)}+\mathrm{V}(i, k),
$$

where $\mathrm{A}(i, k)$ are the modulated data (M-QAM scheme), $\mathrm{H}(i, k)$ represents the channel coefficient affecting sub-carrier $k$ of symbol $i$ and $\mathrm{V}(i, k)$ are the samples of a zero-mean Gaussian noise process, which also includes the ICI.

The phase component $\phi_{i}(k)$ distorting the modulated data $\mathrm{A}(i, k)$ is as follows, considering what it was stated above:

$$
\phi_{i}(k)=\left(m_{i} \cdot k\right)+c_{i}
$$

where the slope $m_{i}$ in symbol $i$ can be expressed as

$$
m_{i}=m_{0}+(i \cdot \xi)
$$

The parameter $\xi$ relates to the sampling clock error and may be positive or negative, depending on whether the sampling is faster or slower than expected. The value $m_{0}$ will be given by an error in the symbol timing estimation and is constant throughout the symbols. The term $c_{i}$ in (2) can be further decomposed for symbol $i$ as

$$
c_{i}=\left(i \cdot c_{0}\right)+\alpha_{i}
$$

where $\alpha_{i}$ is the contribution of the CPE for that particular symbol (random value) and $c_{0}$ is the phase derived from the residual carrier frequency offset, which is accumulated from symbol to symbol .

\section{Channel EstimatoR}

Since pilots cannot be used for channel estimation, a decision-directed channel estimator as shown in Figure 1 was selected. Mignone and Morello firstly proposed this scheme in [5]; however, the use of pilots was completely discarded in their solution.

The interesting point in this channel estimator is that it makes use of a complex divider to correct the data samples (equalizer). The estimator is designed in such a way that the samples of symbol $i$ are used to calculate an estimation of the channel, which will be used to correct the symbol $i+\mathrm{L}$, where $\mathrm{L}$ is the delay introduced by the feedback loop (in symbols). The value $\mathrm{L}$ depends very much on the traceback length of the Viterbi decoder in the FEC block. Since different modulation schemes are being used, a suitable value for the traceback length has to be derived for each of them.

The channel estimation used to correct symbol $i$ will be affected by the phase error of symbol $i-\mathrm{L}$. After division (equalization), the symbol $i$ will retain a residual phase error of the form $\mathrm{D}_{\theta}(i, k) \equiv \mathrm{D}_{k}=\exp \left\{j \cdot\left(\phi_{i}(k)-\phi_{i-\mathrm{L}}(k)\right)\right\}$ with

$$
\phi_{i}(k)-\phi_{i-\mathrm{L}}(k)=\mathrm{L} \cdot \xi \cdot k+\mathrm{L} \cdot c_{0}+\left(\alpha_{i}-\alpha_{i-\mathrm{L}}\right),
$$

which results in a linear residual phase whose slope does not depend on $i$. In the same way, the pilots in symbol $i$ will be divided by the pilots in symbol $i-\mathrm{L}$. In any case, the channel is supposed not to change very much during a period of $\mathrm{L}$ symbols, so that after division, the resulting pilots will be pure phasors with normalized magnitude and a phase given by (5). In the absence of noise, this phasor may be expressed as

$$
\mathrm{P}_{\theta}(i, k) \equiv \mathrm{P}_{k}=e^{j\left(\delta \cdot k+\theta_{i}\right)} ; k=-21,-7,+7,+21
$$

where $\delta=\mathrm{L} \cdot \xi$ and $\theta_{i}=\mathrm{L} \cdot c_{0}+\left(\alpha_{i}-\alpha_{i-\mathrm{L}}\right)$ 


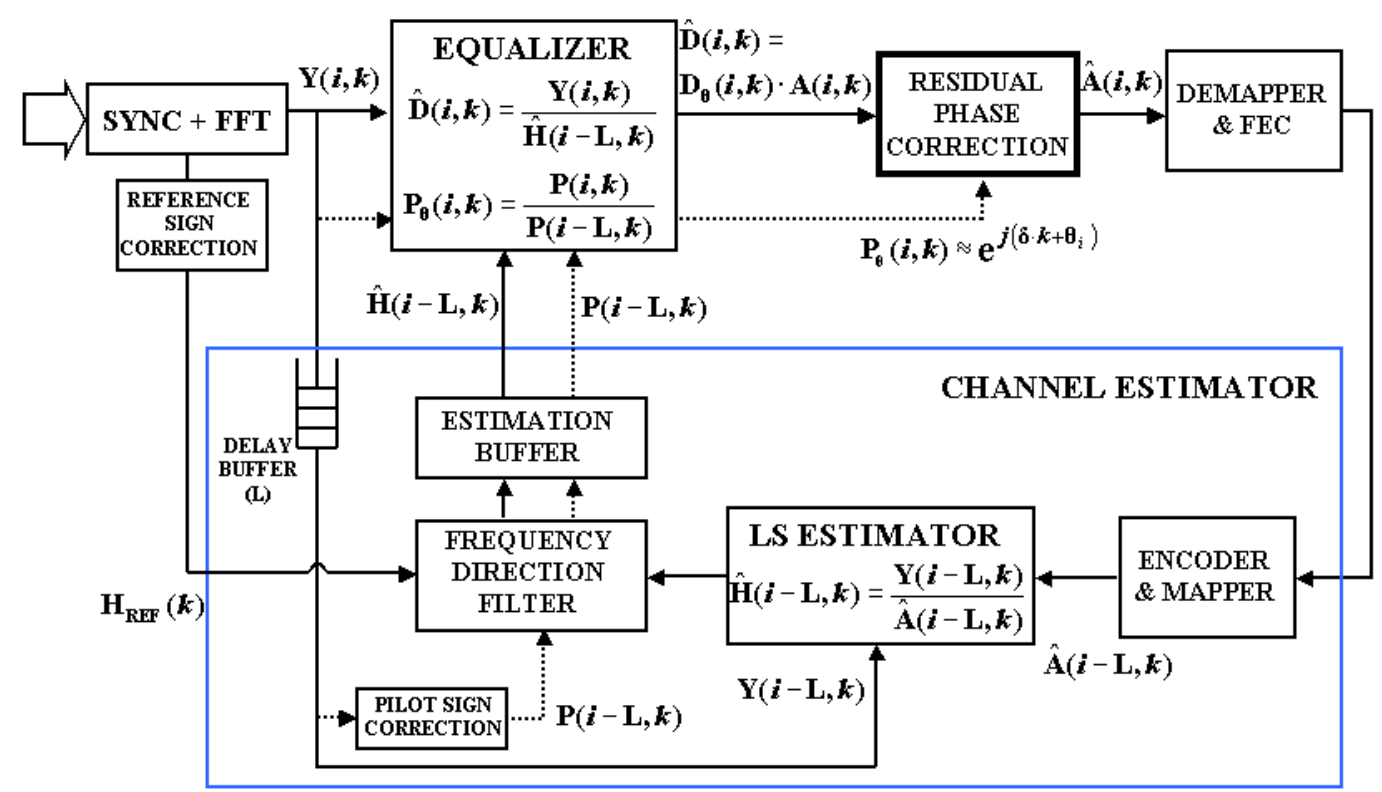

Figure 1. Block diagram of the channel estimator.

\section{RESIDUAL PHASE ERROR: ESTIMATION AND CORRECTION}

The method we propose considers that the condition $|\delta \cdot k|<<1$ is satisfied $\forall k \in[-26,26]$. In this case, the expression in (6) may be simplified by considering a first order approximation of the complex exponential, yielding

$$
\mathrm{P}_{k}=\cos \left(\theta_{i}\right)-\delta \cdot k \cdot \sin \left(\theta_{i}\right)+j\left(\sin \left(\theta_{i}\right)+\delta \cdot k \cdot \cos \left(\theta_{i}\right)\right) \text {. }
$$

At the FFT output (Figure 1), the data are considered to be arranged in the following order: $\mathrm{P}_{-21}, \mathrm{P}_{-7}, \mathrm{P}_{+7}, \mathrm{P}_{+21}, \mathrm{D}_{k}(k=$ $-26, \ldots+26 ; k \neq-21,-7,0,+7,+21)$. The subscript indicates the corresponding sub-carrier position inside the symbol as defined in [1].

In (7) four parameters are of interest: $\cos \left(\theta_{i}\right), \sin \left(\theta_{i}\right)$, $\delta \cdot \sin \left(\theta_{i}\right)$ and $\delta \cdot \cos \left(\theta_{i}\right)$. These parameters can be obtained combining the four available pilots. The former two may be expressed as

$$
\begin{aligned}
& \cos \left(\theta_{i}\right)=(1 / 4)\left(\mathfrak{R}\left\{\mathrm{P}_{-21}\right\}+\mathfrak{R}\left\{\mathrm{P}_{-7}\right\}+\mathfrak{R}\left\{\mathrm{P}_{+7}\right\}+\mathfrak{R}\left\{\mathrm{P}_{+21}\right\}\right) \\
& \sin \left(\theta_{i}\right)=(1 / 4)\left(\mathfrak{I}\left\{\mathrm{P}_{-21}\right\}+\mathfrak{I}\left\{\mathrm{P}_{-7}\right\}+\mathfrak{I}\left\{\mathrm{P}_{+7}\right\}+\mathfrak{I}\left\{\mathrm{P}_{+21}\right\}\right)
\end{aligned}
$$

where $\mathfrak{R}\{$.$\} denotes the real part and \mathfrak{I}\{$.$\} the imaginary part.$ Note that (8) and (9) require no multiplication, but just addition and one shift operation. For the calculation of $\delta \cdot \sin \left(\theta_{i}\right)$, from (7) it is obtained that

$$
35 \cdot \delta \cdot \sin \left(\theta_{i}\right)=(1 / 2)\left(\Re\left\{\mathrm{P}_{-21}\right\}+2 \Re\left\{\mathrm{P}_{-7}\right\}-2 \Re\left\{\mathrm{P}_{+7}\right\}-\Re\left\{\mathrm{P}_{+21}\right\}\right)
$$

28. $\delta \cdot \sin \left(\theta_{i}\right)=(1 / 2)\left(\Re\left\{\mathrm{P}_{-21}\right\}+\Re\left\{\mathrm{P}_{-7}\right\}-\mathfrak{R}\left\{\mathrm{P}_{+7}\right\}-\mathfrak{R}\left\{\mathrm{P}_{+21}\right\}\right)$.

Using the following simplification:

$$
\delta \cdot \sin \left(\theta_{i}\right) \cdot\left(\frac{35}{32}+\frac{28}{32}\right) \approx \delta \cdot \sin \left(\theta_{i}\right) \cdot\left(\frac{36}{32}+\frac{28}{32}\right)=2 \cdot \delta \cdot \sin \left(\theta_{i}\right)
$$

and afterwards, inserting (10.a) and (10.b) into (11), yields

$\delta \cdot \sin \left(\theta_{i}\right)=(1 / 128)\left(2 \Re\left\{\mathrm{P}_{-21}\right\}+3 \Re\left\{\mathrm{P}_{-7}\right\}-3 \Re\left\{\mathrm{P}_{+7}\right\}-2 \mathfrak{R}\left\{\mathrm{P}_{+21}\right\}\right)$.

The simplification done in (11) introduces only an error of $1.6 \%$ into (12). The whole calculation can be still realized using shift operations and additions. Equivalently, $\delta \cdot \cos \left(\theta_{i}\right)$ is obtained as

$\delta \cdot \cos \left(\theta_{i}\right)=-(1 / 128)\left(2 \mathfrak{I}\left\{\mathrm{P}_{-21}\right\}+3 \mathfrak{I}\left\{\mathrm{P}_{-7}\right\}-3 \mathfrak{I}\left\{\mathrm{P}_{+7}\right\}-2 \mathfrak{I}\left\{\mathrm{P}_{+21}\right\}\right)$

Once these four parameters have been determined, the correction of the data sub-channels is straightforward.

The first data after the pilots is $\hat{\mathrm{D}}(i,-26)$. The phase contribution found in this particular sub-carrier due to the residual phase error $\mathrm{D}_{-26}$ can be written as

$$
\mathrm{D}_{-26}=\cos \left(\theta_{i}\right)+26 \cdot \delta \cdot \sin \left(\theta_{i}\right)+j\left(\sin \left(\theta_{i}\right)-26 \cdot \delta \cdot \cos \left(\theta_{i}\right)\right),(14
$$


and so the corresponding sample in the data path should be multiplied by $\left(D_{-26}\right)^{*}$ (complex conjugated) in order to compensate for its residual phase error.

The factors $\delta \cdot \sin \left(\theta_{i}\right)$ and $-\delta \cdot \cos \left(\theta_{i}\right)$ are multiplied by 26 in (14), but actually no multiplication is really needed since $26=$ $2^{5}-2^{2}-2$. Furthermore, this is only necessary for the first sample; for the sample at $k=-25$, the corresponding correcting factor $\left(D_{-25}\right)^{*}$ will be obtained as

$$
\begin{gathered}
\mathfrak{R}\left\{\left(\mathrm{D}_{-25}\right)^{*}\right\}=\mathfrak{R}\left\{\left(\mathrm{D}_{-26}\right)^{*}\right\}-\delta \cdot \sin \left(\theta_{i}\right) \\
\mathfrak{I}\left\{\left(\mathrm{D}_{-25}\right)^{*}\right\}=\mathfrak{I}\left\{\left(\mathrm{D}_{-26}\right)^{*}\right\}-\left(-\delta \cdot \cos \left(\theta_{i}\right)\right) .
\end{gathered}
$$

Special care has to be taken when calculating $\left(D_{-20}\right)^{*}$, $\left(D_{-6}\right)^{*},\left(D_{+1}\right)^{*},\left(D_{+8}\right)^{*},\left(D_{+22}\right)^{*}$. For these particular cases, there is a "hole" left by either the pilots $(k=-21,-7,+7,+21)$ or the DC sub-channel $(k=0)$, thus yielding

$$
\begin{gathered}
\mathfrak{N}\left\{\left(\mathrm{D}_{k}\right)^{*}\right\}=\mathfrak{R}\left\{\left(\mathrm{D}_{k-2}\right)^{*}\right\}-\mathbf{2} \cdot \boldsymbol{\delta} \cdot \sin \left(\theta_{i}\right) \\
\mathfrak{I}\left\{\left(\mathrm{D}_{k}\right)^{*}\right\}=\mathfrak{I}\left\{\left(\mathrm{D}_{k-2}\right)^{*}\right\}-\left(-\mathbf{2} \cdot \boldsymbol{\delta} \cdot \cos \left(\theta_{i}\right)\right),
\end{gathered}
$$

with $k=-20,-6,+1,+8,+22$.

In Figure 2, a block diagram for the implementation of the proposed residual phase correction mechanism is presented. The blocks named $A C C$ carry out the operations in $(15 . \mathrm{a} / \mathrm{b})$ and $(16 . \mathrm{a} / \mathrm{b})$ and are shown in more detail in Figure 3.

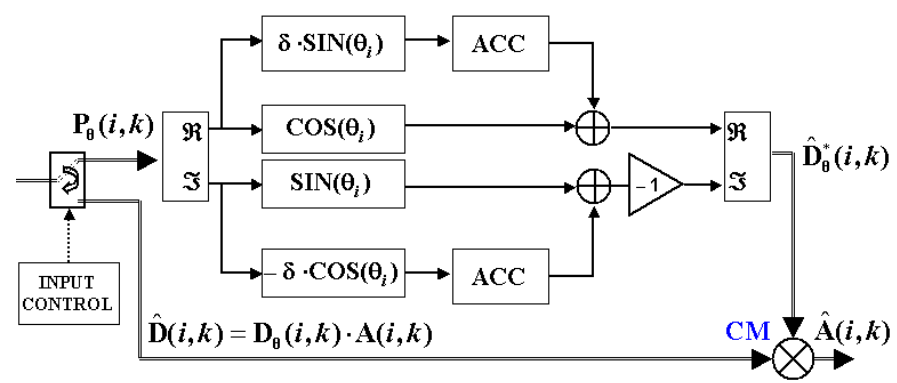

Figure 2. Proposed Residual Phase Correction mechanism.

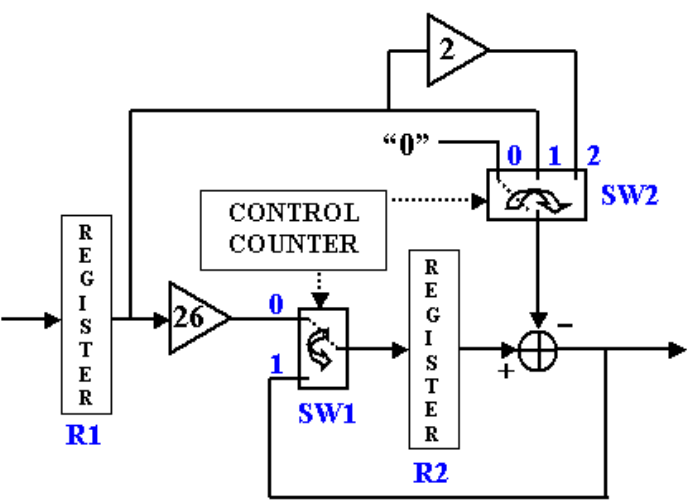

Figure 3. Block diagram of the Accumulators (ACC) shown in Figure 2.
Initially, the register R1 in Figure 3 contains the estimated value for $\delta \cdot \sin \left(\theta_{i}\right)$ (or $\left.-\delta \cdot \cos \left(\theta_{i}\right)\right)$ and the switches SW1 and SW2 are both at position "0". The value of R1 multiplied by 26 will be stored in the register R2. After that, SW1 and SW2 will both switch to position "1". The switch SW2 will only change to position "2" when $\left(\mathrm{D}_{-20}\right)^{*},\left(\mathrm{D}_{-6}\right)^{*},\left(\mathrm{D}_{+1}\right)^{*},\left(\mathrm{D}_{+8}\right)^{*}$ and $\left(D_{+22}\right)^{*}$ are to be calculated. In all the cases, the final correction is achieved by performing a single complex multiplication (block CM in Figure 2).

\section{Simulation Results}

\section{A. AWGN scenario}

The proposed mechanism has been initially simulated in an AWGN scenario, using four different transmission rates: 9, 18, 36 and 54 Mbps as defined in the IEEE 802.11a standard. The SNR was selected to be $20 \mathrm{~dB}$ for the 9 and 18 Mbps cases, 30 $\mathrm{dB}$ for the $36 \mathrm{Mbps}$ case and $40 \mathrm{~dB}$ for the $54 \mathrm{Mbps}$ case. Furthermore, a residual carrier frequency offset after synchronization of $0.4 \%$, i.e. $1.25 \mathrm{kHz}$, was forced in the 9 , 18, and 36 Mbps cases. Due to its higher sensitivity, the 54 Mbps case was simulated with only $0.08 \%$ frequency error, i.e. $250 \mathrm{~Hz}$. A sampling clock offset of $\zeta=80 \mathrm{ppm}\left(\zeta=8 \cdot 10^{-5}\right)$ @20MHz was selected together with a phase noise model as given in [4]. From [3], the parameter $\delta$ in (6) is given by

$$
\delta=\mathrm{L} \cdot \xi=\mathrm{L} \cdot 2 \pi \cdot(4 / 3.2) \cdot \zeta,
$$

being 4/3.2 the ratio between the symbol lengths with and without guard interval.

The traceback length in the Viterbi decoder was selected to be 120 bits long in all the cases. Furthermore, the SIGNAL symbol defined in [1] is not intended for the channel estimation but just for initialization. Hence, according to the number of data bits per OFDM symbol given in [1], this results in the following values for $\mathrm{L}$ : $\mathrm{L}=5$ for $9 \mathrm{Mbps}, \mathrm{L}=3$ for $18 \mathrm{Mbps}, \mathrm{L}=2$ for 36 and $54 \mathrm{Mbps}$. The worst case, i.e. $\mathrm{L}=5$, results in $\delta=5 \cdot 10^{-3}$. The frame length was set to 58 data symbols in all the cases.

The simulation results are shown graphically in Figure 4, where the constellation diagrams before (grey) and after (black) the phase correction are represented.

\section{B. Error Vector Magnitude (EVM) evaluation}

The second test carried out in our simulations consists on the determination of the Error Vector Magnitude (EVM) as defined in [1] for a power-normalized constellation diagram, i.e.

$$
\mathrm{EVM}=\sqrt{\frac{\sum_{i=1}^{\mathrm{L}_{\mathrm{S}}} \sum_{k=1}^{48}\left(\Re\left\{\mathrm{e}_{\mathrm{C}}(i, k)\right\}\right)^{2}+\left(\mathfrak{I}\left\{\mathrm{e}_{\mathrm{C}}(i, k)\right\}\right)^{2}}{48 \cdot \mathrm{L}_{\mathrm{S}}}},
$$


where $\mathrm{L}_{\mathrm{S}}$ states for the number of symbols contained in the frame and $\mathrm{e}_{\mathrm{C}}(i, k)$ is the constellation error given by

$$
\mathrm{e}_{\mathrm{C}}(i, k)=\mathrm{A}(i, k)-\hat{\mathrm{A}}(i, k)
$$

In (18) only the data sub-carriers, i.e. 48 out of 64 , are considered in the calculation of the EVM.

Simulation results are depicted in Figures 5 and 6 for the 9 and $54 \mathrm{Mbps}$ cases, respectively. The results compare the EVM obtained versus the SNR for both AWGN and a fading channel according to the model $\mathrm{A}$ in [6]. For that, groups of 20 frames containing 16 random symbols each were generated. The sampling clock error was $80 \mathrm{ppm}$ in all the cases. The residual carrier frequency offset was $0.4 \%(9 \mathrm{Mbps})$ and $0.08 \%$ (54 Mbps). The traceback length was fixed to 120 bits.

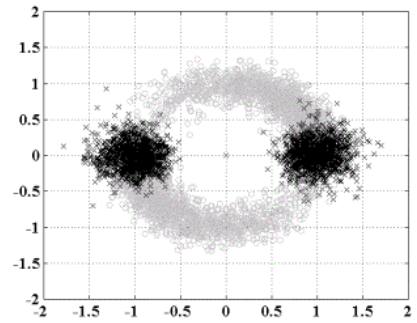

(a)

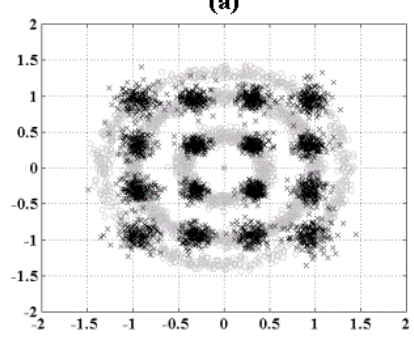

(c)

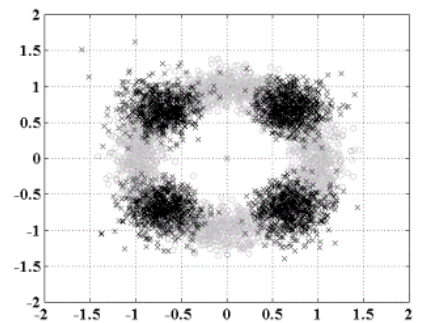

(b)

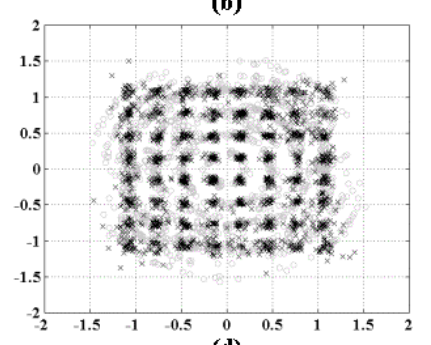

(d)
Figure 4. Simulated constellation diagrams for: (a) $9 \mathrm{Mbps}$; (b) $18 \mathrm{Mbps}$; (c) 36 Mbps; (d) 54 Mbps.

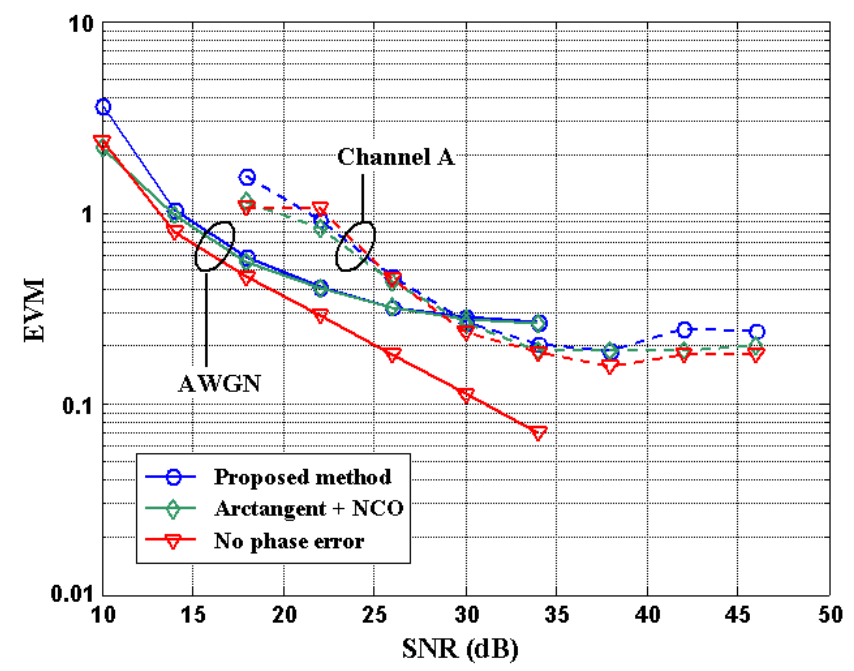

Figure 5. EVM for the 9 Mbps case.

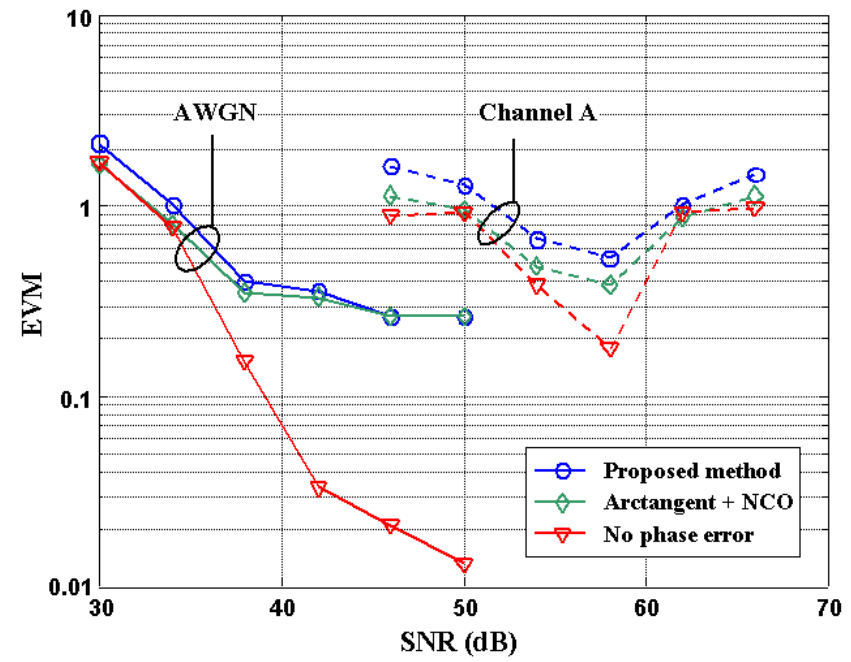

Figure 6. EVM for the 54 Mbps case.

Figures 5 and 6 show that the proposed mechanism does not differ much from the ideal solution based on an arctangent plus NCO, thus simplifying significantly the final implementation. However, in order to reduce the noise content after FFT, a more elaborated solution may include some mechanism to correct for the sampling clock frequency error prior to FFT calculation.

\section{CONCLUSIONS}

A new highly simplified phase tracking mechanism for the IEEE 802.11a standard has been presented in this paper. The method saves a significant amount of hardware, as neither an arctangent block nor an NCO is needed. The latency of the proposed scheme is only one sample, i.e. 50ns @ 20MHz. The validity of this method has been simulated for different transmission rates under pure AWGN and fading conditions. The phase tracking block was programmed in VHDL with a 16-bit fixed-point representation, resulting in a synthesized cell area of only $0.745 \mathrm{~mm}^{2}$ in our in-house $0.25 \mu \mathrm{m}$ BiCMOS technology.

\section{REFERENCES}

[1] IEEE P802.11a/D7.0, Part 11: Wireless LAN Medium Access Control (MAC) and Physical Layer (PHY) specifications: High Speed Physical Layer in the $5 \mathrm{GHz}$ Band, Piscataway, NJ, July 1999.

[2] M. Krstic, A. Troya, K. Maharatna, E. Grass, "Optimized low-power synchronizer design for the IEEE 802.11a standard," Proc. of the IEEE ICASSP'03, vol. II, pp.333-336, Hong Kong, April 6-10 2003.

[3] M. Speth, S.A. Fechtel, G. Fock, and H. Meyr, "Optimum receiver design for wireless broad-band systems using OFDM - part I," IEEE Transactions on Communications, vol. 47, no. 11, pp. 1668-1677, Nov. 1999.

[4] P. Robertson, S. Kaiser, "Analysis of the effects of phase-noise in Orthogonal Frequency Division Multiplex (OFDM) systems," Proc. of the IEEE ICC'95, pp. 1652-1657, June 1995.

[5] V. Mignone, A. Morello, "CD3-OFDM: A novel demodulation scheme for fixed and mobile receivers," IEEE Transactions on Communications, vol. 44, no. 9, pp. 1144-1151, Sept. 1996.

[6] ETSI, "Criteria for comparison," Tech. Rep. 30701F, BRAN PHY Subgroup, May 1998. 